\title{
HLA-B51 subtypes in Turkish patients with Behçet's disease and their correlation with clinical manifestations
}

D.D. Demirseren ${ }^{1}$, G.G. Ceylan ${ }^{2}$, G. Akoglu' ${ }^{1}$, S. Emre' ${ }^{1}$, S. Erten ${ }^{3}$, A. Arman ${ }^{4}$ and A. Metin ${ }^{1}$

${ }^{1}$ Department of Dermatology, Ankara Ataturk Training and Research Hospital, Ankara, Turkey ${ }^{2}$ Department of Medical Genetics, Ankara Ataturk Training and Research Hospital, Ankara, Turkey ${ }^{3}$ Department of Rheumatology, Ankara Ataturk Training and Research Hospital, Ankara, Turkey ${ }^{4}$ Department of Ophthalmology, Ankara Ataturk Training and Research Hospital, Ankara, Turkey

Corresponding author: D.D. Demirseren

E-mail: ddemirseren@yahoo.com

Genet. Mol. Res. 13 (3): 4788-4796 (2014)

Received March 13, 2014

Accepted May 27, 2014

Published July 2, 2014

DOI http://dx.doi.org/10.4238/2014.July.2.8

ABSTRACT. Behçet's disease (BD) is a multisystemic inflammatory disease believed to be triggered by microbial or environmental factors on a genetic platform. Clinically, it may have an impact on many body systems, including the mucocutaneous, ocular, articular, vascular, and neurological systems. In this study, we aimed to determine the HLAB51subtypes and their correlations with the clinical findings of BD. Fiftyone patients with $\mathrm{BD}$ and 44 gender- and age-matched healthy subjects were included in this study. The HLA-B51 subtypes of all participants were determined, and the correlations of the clinical manifestations of the disease with the HLA-B51 subtypes were analyzed. HLA-B51 positivity was found to be significantly higher in the patient group $(\mathrm{P}<$ 
0.001, $R R=15.20$ ), which had significantly more frequent HLA-B5101, HLA-B5102(01), HLA-B5109, and HLA-B5122 subtypes than the healthy subjects (all $\mathrm{P}<0.05$ ). Furthermore, considering the correlation between the genetic makeup and clinical findings, the HLA-B5109 subtype was found to be less frequent in patients with papulopustular skin lesions $(\mathrm{P}=0.042)$. The frequency of HLA-B5103 was significantly higher in patients with central nervous system involvement $(\mathrm{P}=0.015)$. There may be a relationship between HLA-B5102(01), HLA-B5109, and HLA-B5122 in addition to HLA-B51 and HLA-B5101(01) in Turkish patients with BD. The HLA-B5109 subtype can be protective against papulopustular lesion development; however, the HLA-B5103 subtype may pose a risk for neuro-Behçet development in BD.

Key words: Behçet's disease; HLA-B51 subtypes; Clinical correlation

\section{INTRODUCTION}

Behçet's disease (BD) was first defined by the Turkish dermatologist Hulusi Behçet as a triple complex of repetitive aphthous ulcers, genital ulceration, and ocular involvement. Clinically, it is a multisystemic disease that may involve the mucocutaneous, ocular, articular, vascular, intestinal, pulmonary, and neurological systems (Alpsoy et al., 1998). Although the pathogenesis is not exactly known, it is believed to be triggered by microbial or environmental factors, with a genetic predisposition. In many ethnic groups that settled along the ancient Silk Road, a significant relationship is detected between BD and HLA-B51positivity (Kaya et al., 2002; Kötter et al., 2004; de Menthon et al., 2009; Song and Kang, 2012; Hughes et al., 2013; Houman et al., 2013).

The relationship between BD and HLA-B51 subtypes has been investigated in various ethnic groups. These subtypes have shown similarities across ethnic groups, and HLA-B5101 has been identified as the major subtype related with BD. It was claimed that HLA-B5108 is the second most frequent subtype associated with BD (González-Escribano et al., 1998; Kera et al., 1999; Paul et al., 2001; Mizuki et al., 2002; Kötter et al., 2004), and HLA-B5107 was suggested to be negatively correlated with BD. Until now, over 89 HLA-B51 subtypes have been determined in BD (Kaya, 2012), and previous studies have evaluated a maximum of 21 of these HLA-B51 subtypes (Mizuki et al., 2002). There are limited studies on HLA-B51 subtypes in the Turkish population with BD.

In this research, we studied all known HLA-B51 subtypes among BD patients in the Turkish population, and their correlations with the clinical findings. To our knowledge, there is no research evaluating the relationship between HLA-B51subtypes and the clinical findings of BD.

\section{MATERIAL AND METHODS}

\section{Subjects}

Fifty-one patients with Behçet's disease and 44 age- and gender-matched healthy individuals (as the control group) participated in this study. The diagnosis of BD was made accord- 
ing to the criteria of the International Study Group (International Study Group for Behçet's Disease, 1990). Mucocutaneous findings were clinically evaluated by a dermatologist and biopsies were performed when necessary. Patients who had more than three oral ulcers (OUs) per year during their check-ups or in their medical history were regarded as positive for oral ulcers. Patients with active genital ulcers (GUs) or ulcer scars that were identified by dermatologists were regarded as positive for GUs. Pathergy tests were conducted by a dermatologist and evaluated at 24 and $48 \mathrm{~h}$. Erythema nodosum (EN) was diagnosed after clinical evaluation, and when necessary, histopathological investigations were done. Pseudo-folliculitis and acneiform lesions detected in patients who were not on steroid treatment were defined clinically as papulopustular lesions (PPLs) by a dermatologist. Superficial thrombosis was diagnosed through clinical observation, laboratory tests, and Doppler ultrasonography, and ocular involvement was evaluated by an ophthalmologist. Patients with anterior and/or posterior uveitis, visible cells on the vitreous under biomicroscopic evaluation, and vasculitis in the retina were considered as having ocular involvement. Involvement of the joints was evaluated and diagnosed by a rheumatologist. A neuro-Behçet diagnosis was made by a neurologist after neurological examination, magnetic resonance imaging, and microscopic analysis of the cerebrospinal fluid. The presence of paralysis of the cranial nerves and acute meningoencephalitis was regarded as neuro-Behçet. The demographic information and clinical features of all of the patients were recorded.

\section{Determination of HLA-B51 and its subtypes}

Venous blood was obtained from each patient and the control subjects. DNA was isolated from the samples collected (ACD) using a high quality DNA extraction kit, QIAamp DNA Blood Mini blood kit (QIAGEN). The DNA was resuspended in deionized water and stored at $-20^{\circ} \mathrm{C}$ until use for the polymerase chain reaction (PCR). All patients and controls were typed for the HLA-B51 antigen using molecular methods. HLA-B51and the subtypes were determined by PCR using sequence-specific primers (PCR-SSP).

\section{Statistical analyses}

Statistical analyses were performed using the SPSS 15.0 program (Chicago, IL, USA). Normally distributed continuous variables were reported as means \pm standard deviation (means $\pm \mathrm{SD}$ ) and were compared using the Student $t$-test for independent groups. Nonnormally distributed continuous variables were expressed as the median and were compared using the Mann-Whitney U test for independent groups. Categorical variables were reported as numbers and percentages and compared with the chi-square test. A P $<0.05$ was considered to be statistically significant.

This study was approved by the local ethics committee of our hospital and was conducted in accordance with the Declaration of Helsinki.

\section{RESULTS}

Twenty-six patients $(51.0 \%)$ were male and $25(49.0 \%)$ female. The mean age of the patients was $37.51 \pm 11.6$ (range $=19-67$ years). Of these patients, $13.8 \%$ of their first degree family members had BD, and $27.5 \%$ of them had a history of RAS. All patients had OUs, and 
$82.4 \%$ had active GUs or ulcer scars. The presence of GUs was significantly higher in female patients than in the males $(\mathrm{P}=0.011)$.

Thirty-three patients had papulopustular lesions (PPLs), and among the patients with PPLs, 19 (57.57\%) had pseudo-folliculitis and 14 (42.43\%) had acneiform eruptions. The pathergy test was positive in $74.5 \%$ of all patients. Joint involvement was observed in $47 \%$ of the patients, and 4 patients (11.8\%) had neurological involvement; 3 had cranial nerve paralysis and 1 had had acute aseptic meningitis. The demographic and clinical characteristics of the patients are summarized in Table 1.

Table 1. Demographic and clinical characteristics of Behçet's disease (BD) patients.

\begin{tabular}{lc}
\hline Gender, male/female & $26 / 25$ \\
\hline Age (means \pm SD) & $19-67(37.51+-11.6)$ \\
Education (N, \%) & $2(3.9)$ \\
None & $24(47.1)$ \\
Primary school & $8(15.7)$ \\
Intermediate school & $13(25.5)$ \\
Secondary high school & $4(7.8)$ \\
University & $14(27.5)$ \\
Family history of oral ulcer & $7(13.8)$ \\
Family history of BD & $6(11.8)$ \\
Inter-family marriage & $51(100)$ \\
Presence of clinical findings (N, \%) & $42(82.4)$ \\
Oral ulcers & $38(74.5)$ \\
Genital ulcers & $33(64.7)$ \\
Pathergy positivity & $18(35.3)$ \\
PPLs & $18(35.3)$ \\
EN & $24(47.1)$ \\
Uveitis & $6(11.8)$ \\
Articular involvements & $4(7.8)$ \\
Thrombosis & $36(70.6)$ \\
Central nervous system & \\
HLA-B51(+) &
\end{tabular}

$\mathrm{PPL}=$ papulopustular; $\mathrm{EN}=$ erythema nodosum.

In the patient group, HLA-B51 was significantly more frequent than in the control subjects $(P<0.001, R R=15.20)$. The frequency of HLA-B51 positivity was significantly higher in the male $\mathrm{BD}$ patients than in the female ones $(\mathrm{P}=0.039)$. When the HLA-B51subtypes were evaluated, the HLA-B5101, HLA-B5102(01), HLA-B5109, and HLA-B5122 subtypes were determined to be more frequent than in the healthy subjects $(\mathrm{P}<0.001,0.015,0.043$, and 0.003 , respectively) (Table 2). When the data from the patient and control groups were compared in the HLAB51-positive group only, there was no statistically significant difference $(\mathrm{P}>0.05$ for all). Table 3 summarizes the HLA-B51 subtypes and their positive frequency in the patient and control groups.

Table 2. Comparison of frequency of HLA-B 51 subtypes Behçet's disease (BD) and control subjects.

\begin{tabular}{lcccc}
\hline & BD (\%) & Control (\%) & Relative risk \\
& $\mathrm{N}=51$ & $\mathrm{~N}=44$ & $(95 \%$ confidence interval) & P \\
\hline B51 & $36(70.6)$ & $6(13.6)$ & $15.20(5.31-43.47)$ & $<0.01$ \\
B5101 & $35(68.6)$ & $6(13.6)$ & $13.85(4.87-39.37)$ & $<0.01$ \\
B5101(01) & $33(67.9)$ & $6(13.6)$ & $3.61(4.12-32.68)$ & $<(1.30-11.69)$ \\
B5102(01) & $17(33.3)$ & $5(11.7)$ & $3.76(0.97-14.48)$ & 0.015 \\
B5109 & $11(21.5)$ & $3(6.8)$ & $0.48(0.39-0.60)$ & 0.043 \\
B5122 & $9(17.6)$ & $0(0.0)$ & & 0.003 \\
\hline
\end{tabular}


Table 3. Frequency of HLA-B51(+) in Behçet's disease (BD) and control subject's subtypes.

\begin{tabular}{lccc}
\hline & BD (\%) & Control (\%) & Total HLA-B51(+) population \\
& $\mathrm{N}=36$ & $\mathrm{~N}=6$ & $\mathrm{~N}=42$ \\
\hline B5101 & $35(97.2)$ & $6(100)$ & $41(97.6)$ \\
B5101(01) & $33(91.6)$ & $6(100)$ & $39(92.8)$ \\
B5102(01) & $17(47.2)$ & $5(83.3)$ & $22(52.4)$ \\
B5103 & $9(25.0)$ & $3(50.0)$ & $12(28.6)$ \\
B5106 & $1(2.7)$ & $0(0.0)$ & $1(2.4)$ \\
B5107 & $1(2.7)$ & $1(16.6)$ & $2(4.8)$ \\
B5108 & $4(11.1)$ & $1(16.6)$ & $5(11.9)$ \\
B5109 & $11(30.5)$ & $3(50.0)$ & $14(33.3)$ \\
B5111 & $8(22.2)$ & $2(33.3)$ & $10(2.4)$ \\
B5112 & $2(5.5)$ & $1(16.6)$ & $3(7.1)$ \\
B5113 & $2(5.5)$ & $1(16.6)$ & $3(7.1)$ \\
B5114 & $4(11.1)$ & $1(16.6)$ & $5(11.9)$ \\
B5119 & $6(16.6)$ & $1(33.3)$ & $8(19.0)$ \\
B5120 & $1(2.7)$ & $6(10.6)$ & $2(4.8)$ \\
B5121 & $6(16.6)$ & $0(0.0)$ & $12(28.5)$ \\
B5122 & $9(25.0)$ & $2(33.3)$ & $9(21.4)$ \\
B5123 & $6(16.6)$ & $0(0.0)$ & $8(19.0)$ \\
B5124 & $2(5.5)$ & $0(0.0)$ & $2(4.8)$ \\
B5128 & $1(2.7)$ & $0(0.0)$ & $1(2.4)$ \\
B5137 & $3(8.3)$ & $1(16.6)$ & $3(7.1)$ \\
B5144 & $1(2.7)$ & $1(16.6)$ & $2(4.8)$ \\
B5149 & $0(0.0)$ & $0(0.0)$ & $1(2.4)$ \\
B5159 & $2(5.5)$ & $1(16.6)$ & $2(4.8)$ \\
B5163 & $2(5.5)$ & $0(0.0)$ & $3(7.1)$ \\
B5177 & $1(2.7)$ & & $1(2.4)$ \\
\hline
\end{tabular}

With regard to the correlation between the clinical findings of BD and the HLA-B51 subtypes, HLA-B5109 was significantly less frequent in the patients with PPLs ( $\mathrm{P}=0.042)$. Furthermore, HLA-B5103 showed a significantly higher frequency in the patients with neurological involvement $(\mathrm{P}=0.015)$ (Table 4).

Tablo 4. Comparison of frequency of clinic manifestation and HLA-B 51 subtypes.

\begin{tabular}{|c|c|c|c|c|c|c|}
\hline$\overline{\text { HLA }}$ & $\begin{array}{c}\text { B5103(+) } \\
\mathrm{N}=9\end{array}$ & $\begin{array}{c}\text { B5103(-) } \\
\mathrm{N}=42\end{array}$ & $P$ & $\begin{array}{c}\text { B5109(+) } \\
\mathrm{N}=11\end{array}$ & $\begin{array}{c}\text { B5109(-) } \\
\mathrm{N}=40\end{array}$ & $P$ \\
\hline Genital ulcers & $8(88.9)$ & $34(81.0)$ & 1.00 & $8(72.7)$ & $34(85.0)$ & 0.38 \\
\hline Erythema nodosum & $3(33.3)$ & $15(35.7)$ & 1.00 & $4(36.4)$ & $14(35.0)$ & 1.00 \\
\hline Eye involvement & $2(22.2)$ & $6(38.1)$ & 0.46 & $6(54.5)$ & $12(30.0)$ & 0.16 \\
\hline Pathergy positivity & $6(66.7)$ & $27(64.3)$ & 1.00 & $8(72.7)$ & $25(62.5)$ & 0.72 \\
\hline PPLs & $5(55.6)$ & $19(45.2)$ & 0.71 & $2(18.2)$ & $22(55.0)$ & 0.04 \\
\hline Trombosis & $1(11.1)$ & $5(11.9)$ & 1.00 & $1(9.1)$ & $5(12.5)$ & 1.00 \\
\hline Articular involvement & $5(55.6)$ & $33(78.6)$ & 0.20 & $9(81.8)$ & $29(72.5)$ & 0.70 \\
\hline CNS involvement & $3(33.3)$ & $1(2.4)$ & 0.015 & $2(18.2)$ & $2(5.0)$ & 0.19 \\
\hline
\end{tabular}

$\mathrm{PPL}=$ papulopustular lesion; $\mathrm{CNS}=$ central nervous system.

\section{DISCUSSION}

BD is a systemic inflammatory disease, and its etiology is not entirely known. It is believed to be strongly related to a genetic predisposition, depending on its frequency in a specific geographical area, its close relationship with HLA-B51 in different ethnic groups, and familial distribution. HLA-B51gene positivity is frequently seen in areas where Turkish tribes immigrated along the Silk Road, which seems to support the genetic influence (Azizlerli et al., 2003, Papoutsis et al., 2006; Varol et al., 2010; Hamzaoui et al., 2012). 
The contribution of HLA-B51 positivity to BD is approximately $20 \%$, and was indicated as $40-80 \%$ among different ethnic groups. In our study, HLA-B51 positivity was found to be $70.6 \%$, which was compatible with other studies (Kaya, 2012; Maldini et al., 2012). Furthermore, we found that the frequency of HLA-B51 was higher in male patients. This finding is consistent with earlier studies on HLA-B5/B51 positivity. (de Menthon et al., 2009; Maldini et al., 2012).

The major histocompatibility complex (MHC) is the gene region that encodes the tissue antigens that allow the immune system to distinguish between foreign substances and self substances. Human leukocyte antigen (HLA) is the area in humans that carries these tissue antigens, and it resides on chromosome 6. HLA is related to many genes, playing a role in immune response and inflammation, and it shows significant polymorphism, taking part in the pathogenesis of systemic autoimmune diseases. MHC alleles, usually inherited as a unit, do not show a random distribution on individual chromosomes; some tend to occur together much more or less often than predicted by their frequencies. This is called linkage disequilibrium (LD) (Alpsoy et al., 1998; Hughes et al., 2013). Whether the relationship between BD and HLA-B51 takes place directly with the gene itself or due to LD has been a mystery to scientists for a long time.

In many populations, HLA-B5101 has been identified as the major subtype of HLA-B51 (González-Escribano et al., 1998; Mizuki et al., 2001; Paul et al., 2001). HLA-B5101 positivity has been reported to be $62-98 \%$ in various populations (Suzuki and Suzuki, 2004). In our study, we identified HLA-B5101 as the most common subtype (97.2\%) among all of the HLA-B51 subtypes. Two of the patients who had HLA-B5101 had HLA-B510108 (5.7\%), and the other patients had HLA-B510101 (94.3\%). A study has shown that the gene responsible for BD is the HLA-B51 itself, and that there is no polymorphism in the gene that causes the disease (Sano et al., 2001). A study of HLA-B510101 in Turkish, Jordanian, Japanese, and Iranian BD patients found that the gene was the same across all ethnic groups; additionally, predisposition to BD was not caused by any LD or other subtype, but by the HLA-B510101 itself (Takemoto et al., 2008).

In our study, the second most detected subtype across all BD patients was HLAB5102(01) (33.3\%). Among the HLA-B51-positive patients, HLA-B510201 positivity increased to $47.2 \%$. Individuals in the control group had an HLA positivity of $11.7 \%$, while in the patient group, positivity was as high as $83.3 \%$. Until now, there have been no studies indicating HLA-B510201 positivity in Turkish patients with BD. One study has demonstrated the lack of predisposition to $\mathrm{BD}$, even though the HLA-B51 positivity rate is high in the Amerindian population, with the fact that the HLA-B510201positivity rate is high in this population (Piga and Mathieu, 2011). However, in our study, we found that Turkish patients with BD have a high HLA-B510201 allele frequency, which suggests that genetic predisposition to BD is not the only factor in the etiology, and that environmental factors are also influential (Galeone et al., 2012).

HLA-B5108 allele positivity was found to be $10-30 \%$ in various populations. In Middle Eastern, Italian, Spanish, Greek, Japanese, Turkish, and German populations, HLA-B5108 is also suggested to be related to BD (González-Escribano et al., 1998; Kera et al., 1999; Paul et al., 2001; Mizuki et al., 2002; Kötter et al., 2004). In our study, we identified HLA-B5108 positivity as $4 \%$. HLA-B5108 positivity was detected in four patients and one healthy subject from the control group, but no significant difference was detected between the two groups.

In a study where the HLA-B51 subtypes were analyzed in Turkish and German patients, it was suggested that HLA-B5107 could be negatively correlated with the disease (Kötter et al., 2004). In our study, one individual in the patient group and another in the control group were positive for HLA-B5107, and no significant difference was detected between the two groups. 
The third most frequently detected subtype in our patients was HLA-B5109. It has not been previously suggested that this gene is frequent in BD. Of the patient group, $11(21.5 \%)$ were positive for HLA-B5109, and this positivity was significantly higher when compared to the control group. Furthermore, 9 of the patients (17.6\%) were positive for HLA-B5122; however, we did not detect HLA-B5122 positivity in any patient in the control group.

Considering the clinical findings in the BD patients, $100 \%$ had OUs, $82.4 \%$ had GUs, $47.1 \%$ had joint involvement, and $35.3 \%$ had ocular involvement and EN, consistent with previous studies (Tursen et al., 2003; Alpsoy et al., 2007, 2012). The GUs were more frequent in the female patients, which is consistent with an earlier study, in which the clinical findings of 2313 patients were analyzed (Tursen et al., 2003). The frequency of pathergy positivity in patients living in Mediterranean countries (84-98\%) was suggested to be higher than the patients living in Far East (40-70\%) (Lee et al., 1997; Alpsoy et al., 1998; Varol et al., 2010). The pathergy test was positive in $74.5 \%$ of our patients, which is higher than the results of other authors $(56.1 \%)$ (Tursen et al., 2003; Alpsoy et al., 2007).

PPL is one of the frequent clinical findings in BD, and PPLs were observed in $64.7 \%$ of our patients. The frequency of PPLs was found to be 55.4 and $54.0 \%$ in two other study populations (Tursen et al., 2003; Alpsoy et al., 2007). When the subtypes were analyzed, PPLs were significantly lower in the HLA-B5109-positive patients. We suggest that HLA-B5109 positivity may be a protective factor against PPLs in patients with BD.

In BD patients, mortality is frequently related to pulmonary artery involvement, neurological involvement, and intestinal perforation (Tursen et al., 2003). Although gastrointestinal system involvement and major vascular involvement were not detected in our patients, 4 had neurological involvement. Due to a lack of valid diagnostic criteria and limited patient participation in research studies, little is known regarding neurological involvement in BD. The prevalence of neurological involvement in BD is reported as 5-15\%. Typical presentations include focal parenchymal lesions, vascular thrombosis, arterial vasculitis, and aseptic meningoencephalitis (Houman et al., 2013). In our study, neurological involvement was detected in $7.8 \%$ of patients. Although the patients with neuro-Behçet disease were very few, the significant HLA-B5103 positivity caught our attention, and we wonder whether it may indicate a predisposition to neurological involvement in $\mathrm{BD}$.

\section{CONCLUSION}

Although the small sizes of the patient and control groups were a limitation in our study, there may be a relationship between HLA-B5102(01), HLA-B5109, and HLA-B5122, in addition to HLA-B51 and HLA-B5101(01), in Turkish patients with BD. The frequency of these subtypes differs from that of other subtypes, with the exception of the most frequent subtype, HLA-B5101.

Since a negative correlation exists between PPL involvement and the HLA-B5109 suballele, this subtype may be protective against PPLs. Additionally, HLA-B5103 may be a risk factor for neuro-Behçet in Turkish patients with BD.

\section{Conflicts of interest}

The authors declare no conflict of interest. 


\title{
ACKNOWLEDGMENTS
}

\author{
We would like to thank Elife Öztürk for her contributions.
}

\section{REFERENCES}

Alpsoy E (2012). New evidence-based treatment approach in Behçet's disease. Patholog. Res. Int. 2012:871019. Available at [http://www.hindawi.com/journals/pri/2012/871019/] Accessed December 2013.

Alpsoy E, Yilmaz E, Coskun M, Savas A, et al. (1998). HLA antigens and linkage disequilibrium patterns in Turkish Behçet's patients. J. Dermatol. 25: 158-162.

Alpsoy E, Donmez L, Onder M, Gunasti S, et al. (2007). Clinical features and natural course of Behçet's disease in 661 cases: a multicentre study. Br. J. Dermatol. 157: 901-906.

Azizlerli G, Kose AA, Sarica R, Gul A, et al. (2003). Prevalence of Behçet's disease in Istanbul, Turkey. Int. J. Dermatol. 42: 803-806.

de Menthon M, Lavalley MP, Maldini C, Guillevin L, et al. (2009). HLA-B51/B5 and the risk of Behçet's disease: a systematic review and meta-analysis of case-control genetic association studies. Arthritis Rheum. 61: 1287-1296.

Galeone M, Colucci R, D’Erme AM and Moretti S (2012). Potential Infectious Etiology of Behçet’s Disease . Patholog. Res. Int. 2012:595380. doi: 10.1155/2012/595380. Available at [http://www.hindawi.com/journals/pri/2012/595380/]. Accessed December 2013.

González-Escribano MF, Rodríguez MR, Walter K, Sanchez-Roman J, et al. (1998). Association of HLA-B51 subtypes and Behçet's disease in Spain. Tissue Antigens 52: 78-80.

Hamzaoui A, Houman MH, Massouadia M, Ben ST, et al. (2012). Contribution of Hla-B51 in the susceptibility and specific clinical features of Behçet's disease in Tunisian patients. Eur. J. Intern. Med. 23: 347-349.

Houman MH, Bellakhal S, Ben ST, Hamzaoui A, et al. (2013). Characteristics of neurological manifestations of Behçet's disease: a retrospective monocentric study in Tunisia. Clin. Neurol. Neurosurg. 115: 2015-2018.

Hughes T, Coit P, Adler A, Yilmaz V, et al. (2013). Identification of multiple independent susceptibility loci in the HLA region in Behçet's disease. Nat. Genet. 45: 319-324.

International Study Group for Behçet's Disease (1990). Criteria for diagnosis of Behçet's disease. Lancet 335: 1078-1080.

Kaya TI (2012). Genetics of Behçet's Disease. Patholog. Res. Int. Pathology Research International, vol. 2012, 6. Available at [http://www.hindawi.com/journals/pri/2012/912589/]. Accessed December 2013.

Kaya TI, Dur H, Tursen U and Gurler A (2002). Association of class I HLA antigens with the clinical manifestations of Turkish patients with Behçet's disease. Clin. Exp. Dermatol. 27: 498-501.

Kera J, Mizuki N, Ota M, Katsuyama Y, et al. (1999). Significant associations of HLA-B*5101 and B*5108, and lack of association of class II alleles with Behçet's disease in Italian patients. Tissue Antigens 54: 565-571.

Kötter I, Vonthein R, Muller CA, Gunaydin I, et al. (2004). Behçet's disease in patients of German and Turkish origin living in Germany: a comparative analysis. J. Rheumatol. 31: 133-139.

Lee ES, Bang D and Lee S (1997). Dermatologic manifestation of Behçet's disease. Yonsei Med. J. 38: 380-389.

Maldini C, Lavalley MP, Cheminant M, de Menthon M, et al. (2012). Relationships of HLA-B51 or B5 genotype with Behçet's disease clinical characteristics: systematic review and meta-analyses of observational studies. Rheumatology 51: 887-900.

Mizuki N, Ota M, Katsuyama Y, Yabuki K, et al. (2001). HLA-B*51 allele analysis by the PCR-SBT method and a strong association of HLA-B*5101 with Japanese patients with Behçet's disease. Tissue Antigens 58: 181-184.

Mizuki N, Ota M, Katsuyama Y, Yabuki K, et al. (2002). Sequencing-based typing of HLA-B*51 alleles and the significant association of HLA-B*5101 and -B*5108 with Behçet's disease in Greek patients. Tissue Antigens 59: 118-121.

Papoutsis NG, Abdel-Naser MB, Altenburg A, Orawa H, et al. (2006). Prevalence of Adamantiades-Behçet's disease in Germany and the municipality of Berlin: results of a nationwide survey. Clin. Exp. Rheumatol. 24: S125.

Paul M, Klein T, Krause I, Molad Y, et al. (2001). Allelic distribution of HLA-B*5 in HLA-B5-positive Israeli patients with Behçet's disease. Tissue Antigens 58: 185-186.

Piga M and Mathieu A (2011). Genetic susceptibility to Behçet's disease: role of genes belonging to the MHC region. Rheumatology 50: 299-310.

Sano K, Yabuki K, Imagawa Y, Shiina T, et al. (2001). The absence of disease-specific polymorphisms within the HLA-B51 gene that is the susceptible locus for Behçet's disease. Tissue Antigens 58: 77-82.

Song YW and Kang EH (2012). Behçet's disease and genes within the major histocompatibility complex region. Mod. Rheumatol. 22: 178-185. 
Suzuki KM and Suzuki N (2004). Behçet's disease. Clin. Exp. Med. 4: 10-20.

Takemoto Y, Naruse T, Namba K, Kitaichi N, et al. (2008). Re-evaluation of heterogeneity in HLA-B*510101 associated with Behçet's disease. Tissue Antigens 72: 347-353.

Tursen U, Gurler A and Boyvat A (2003). Evaluation of clinical findings according to sex in 2313 Turkish patients with Behçet's disease. Int. J. Dermatol. 42: 346-351.

Varol A, Seifert O and Anderson CD (2010). The skin pathergy test: innately useful?. Arch. Dermatol. Res. 302: 155-158. 